Commun. math. Phys. 10, 155-178 (1968)

\title{
On the Existence of a Local Hamiltonian in the Galilean Invariant Lee Model
}

\author{
R. SCHRADER
}

Seminar für theoretische Physik der E. T. H., Zürich

Received May 10, 1968

\begin{abstract}
It is shown that there exists a selfadjoint Hamilton operator in the limit of local coupling for the Galilean invariant Lee Model. We discuss the scattering theory of this Hamilton operator in the $V \Theta-N \Theta \Theta$ sector.
\end{abstract}

\section{§1. Introduction}

Recently J. M. Levy-LeBlond [1] has discussed properties of Galilean invariant field theories. Although one has the Bargmann superselection rule for the mass [2], nevertheless such theories may describe processes involving particle creation and annihilation. In particular J. M. Levy-LeBLond has given a Galilean invariant formulation of the Lee Model [3]. In its original form the Lee Model has been the object of great interest. It is solvable in the lowest sectors [4] and there is a mass and coupling constant renormalization. The Tamm-Dancoff method [5] has been applied as well as the LSZ-formalism [6] and dispersion relation methods [7] have also been used. However, it was always necessary to use a cutoff function and to consider possible ghost states.

The Galilean invariant formulation also describes the interaction of three particles $V, N$ and $\Theta ; V \leftrightarrow N+\Theta$ being the possible transitions. The free particle theory is given by 3 fields $V(P), \Theta(k), N(l)$ satisfying the following (anti-)commutation relations

$$
\begin{gathered}
\left\{V(P), V^{*}\left(P^{\prime}\right)\right\}=\delta^{3}\left(P-P^{\prime}\right) ;\left\{V(P), V\left(P^{\prime}\right)\right\}=0 \\
{\left[\Theta(k), \Theta^{*}\left(k^{\prime}\right)\right]=\delta^{3}\left(k-k^{\prime}\right) ;\left[\Theta(k), \Theta\left(k^{\prime}\right)\right]=0} \\
\left\{N(l), N^{*}\left(l^{\prime}\right)\right\}=\delta^{3}\left(l-l^{\prime}\right) ;\left\{N(l), N\left(l^{\prime}\right)\right\}=0 \text { etc. }
\end{gathered}
$$

The Hilbert space is the Fock space defined by these fields. The free 1-particle $V$-states transform according to an irreducible representation of the central extension of the Galilei group with mass $m_{1}$, spin 0 and internal energy $U_{0}[2,8]$.

The masses of the $\Theta$ and $N$ particles are $m_{2}$ and $m_{3}$ respectively, their spin and their internal energy is zero. $V$ and $N$ are fermions; $\Theta$ is a boson; but the choice of statistics is not important [1]. The free 
Hamiltonian therefore becomes

$$
\begin{aligned}
H_{0}\left(U_{0}\right) & =\int\left(U_{0}+\frac{P^{2}}{2 m_{1}}\right) V^{*}(P) V(P) d^{3} P \\
& +\int \frac{k^{2}}{2 m_{2}} \Theta^{*}(k) \Theta(k) d^{3} k+\int \frac{l^{2}}{2 m_{3}} N^{*}(l) N(l) d^{3} l .
\end{aligned}
$$

The interaction is defined as

$$
\begin{aligned}
H_{I \chi} & =\lambda_{0} \int \chi(\omega)\left[V^{*}(P) N\left(\frac{m_{3}}{m_{1}} P+q\right) \Theta\left(\frac{m_{2}}{m_{1}} P-q\right)+h \cdot c\right] d^{3} P d^{3} q \\
2 \mu \omega & =q^{2} ; \quad \mu m_{1}=m_{2} m_{3} .
\end{aligned}
$$

Bargmann's superselection rule requires $m_{1}=m_{2}+m_{3} . \lambda_{0}$ is the coupling constant and $\chi$ is a real cut-off function. We will assume $\chi$ to be smooth with compact support and $0 \leqq \chi \leqq 1$. Then $H_{\chi}=H_{0}\left(U_{0}\right)+H_{I \chi}$ will be a selfadjoint Hamilton operator. More precisely: Let $\mathscr{N}(V)$, $\mathscr{N}(\Theta), \mathscr{N}(N)$ be the three particle number operators. Then $\mathscr{N}_{1}=\mathscr{N}(V)$ $+\mathscr{N}(\Theta)$ and $\mathscr{N}_{2}=\mathscr{N}(V)+\mathscr{N}(N)$ are constants of motion. The mass operator is $\mathscr{M}=m_{2} \mathscr{N}_{1}+m_{3} \mathscr{N}_{2}$. Let $\mathscr{H}\left(N_{1}, N_{2}\right)\left(N_{1}, N_{2}\right.$ nonnegative integers) be the sector corresponding to the eigenvalues $N_{1}$ and $N_{2}$ of $\mathscr{N}_{1}$ and $\mathscr{N}_{2}$. Since all particle number operators are bounded in $\mathscr{H}\left(N_{1}, N_{2}\right)$, $H_{I \chi}$ restricted to each sector is a bounded self-adjoint operator. Then $H_{\chi}$ is selfadjoint in $\mathscr{H}\left(N_{1}, N_{2}\right)$ and the domains of definition of $H_{\chi}$ and $H_{0}\left(U_{0}\right)$ coincide in each sector [9]. Let $(2 \mathscr{M})^{-1} \mathscr{P}^{2}$ be the center of mass energy operator and put $H_{\chi}{ }^{S}=H_{\chi}-(2 \mathscr{M})^{-1} \mathscr{P}^{2}$. Considering $\mathscr{H}(1,1)$ we may write this space as $\mathscr{H}_{1} \otimes \mathscr{H}_{2}$, where $\mathscr{H}_{1}$ is the Hilbertspace of the center of mass motion and $\mathscr{H}_{2}$ consists of all pairs $(g, f)$ with $g \in \mathbb{C}$ and $f \in \mathscr{L}^{2}\left(\mathbb{R}^{3}\right) . H_{\chi}{ }^{S}$ restricted to $\mathscr{H}(1,1)$ then only acts on $\mathscr{H}_{2}$. If we write the resolvent $r^{S}(\chi, z)$ of $H_{\chi}{ }^{S}$ as a $2 \times 2$-matrix, then its kernel has the form [1]:

$r^{S}(\chi, z)\left(q, q^{\prime}\right)$

$$
=\left(\begin{array}{c}
\frac{Z_{\chi}}{H(\chi, z-U)} ; \frac{\lambda Z_{\chi}^{1 / 2} \chi\left(\omega^{\prime}\right)}{H(\chi, z-U)\left(z-\omega^{\prime}\right)} \\
\frac{\lambda Z_{\chi}^{1 / 2} \chi(\omega)}{(z-\omega) H(\chi, z-U)} ; \frac{\delta^{3}\left(q-q^{\prime}\right)}{z-\omega}+\frac{\lambda^{2} \chi(\omega) \chi\left(\omega^{\prime}\right)}{(z-\omega) H(\chi, z-U)\left(z-\omega^{\prime}\right)}
\end{array}\right)
$$

where

$$
\begin{aligned}
Z_{\chi}^{-1} & =1+\lambda_{0}^{2} \int \frac{\chi^{2}(\omega) d^{3} q}{(U-\omega)^{2}}=1+\lambda_{0}^{2} \lambda_{c}^{-2}(\chi) \\
U_{0} & =1-\lambda_{0}^{2} \int \frac{\chi^{2}(\omega) d^{3} q}{(U-\omega)}=U+\delta U_{\chi} \\
\lambda & =Z_{\chi}^{1 / 2} \lambda_{0} .
\end{aligned}
$$

The function $H(\chi, z)$ is discussed in Appendix A. $U$ is required to be smaller than zero. $r^{S}(\chi, z)$ has a pole at $z=U$ and a cut $0 \leqq z<\infty$. 
Let $e^{\prime}(\chi), \Delta(\tau), r_{0}{ }^{S}(z), r_{0}{ }^{R}, S(\chi, z)$ and $t(\chi, z)$ be defined by

$$
\begin{aligned}
e^{\prime}(\chi)\left(q, q^{\prime}\right)=\left(\begin{array}{cc}
Z_{\chi}^{1 / 2} & 0 \\
0 & \delta^{3}\left(q-q^{\prime}\right)
\end{array}\right) ; \Delta(\tau)\left(q, q^{\prime}\right) & =\left(\begin{array}{cc}
\delta(\tau-U) & 0 \\
0 & \delta(\tau-\omega) \delta^{3}\left(q-q^{\prime}\right)
\end{array}\right) \\
r_{0}^{S}(z)\left(q, q^{\prime}\right) & =\left(\begin{array}{cc}
\frac{1}{z-U} & 0 \\
0 & \frac{\delta^{3}\left(q-q^{\prime}\right)}{z-\omega}
\end{array}\right) ; \\
r_{0}{ }^{R, S}(\chi, z)\left(q, q^{\prime}\right) & =\left(\begin{array}{cc}
\frac{Z_{\chi}^{1 / 2}}{H(\chi, z-U)} & 0 \\
0 & \frac{\delta^{3}\left(q-q^{\prime}\right)}{z-\omega}
\end{array}\right) ; \\
t(\chi, z)\left(q, q^{\prime}\right) & =\left(\begin{array}{cc}
0 & \lambda \chi\left(\omega^{\prime}\right) \\
\lambda \chi(\omega) ; \frac{\lambda^{2} \chi(\omega) \chi\left(\omega^{\prime}\right)}{H(\chi, z-U)}
\end{array}\right) .
\end{aligned}
$$

Then the scattering operators $u^{ \pm}(\chi)$ are given by

$$
u^{ \pm}(\chi)=\int_{-\infty}^{+\infty}\left(e^{\prime}+r_{0} R, S(\chi, \tau \mp i 0) t(\chi, \tau \mp i 0)\right) \Delta(\tau) d \tau .
$$

$u^{ \pm}(\chi)$ are defined on a dense set in $\mathscr{H}_{2}$ consisting of all pairs $(g, f)$ where $f$ is smooth with compact support. On this set

$$
\left(u^{ \pm}(\chi)\right)^{\dagger} u^{ \pm}(\chi)=\mathbf{1}
$$

so $u^{ \pm}(\chi)$ may be extended to isometric operators on $\mathscr{H}_{2}$. Furthermore unitarity holds :

and also

$$
u^{ \pm}(\chi)\left(u^{ \pm}(\chi)\right)^{\dagger}=\mathbf{1}
$$

$$
r^{S}(\chi, z) u^{ \pm}(\chi)=u^{ \pm}(\chi) r_{0}^{S}(z) .
$$

Since $r_{0}{ }^{S}(z)$ is the resolvent of $H_{0}{ }^{S}(U)=H_{0}(U)-(2 \mathscr{M})^{-1} \mathscr{P}^{2}$, we see that $u^{ \pm}(\chi)$ are indeed intertwining operators for $H_{\chi}{ }^{S}$ and $H_{0} S(U) . U$ is therefore the renormalized internal energy, $Z_{z}$ is the renormalization constant and $\lambda$ the renormalized coupling constant. We will keep $U<0$ and $\lambda$ fixed, so that $U_{0}=U_{0}(\chi) ; \lambda_{0}=\lambda_{0}(\chi)$. Then $\lambda$ has to satisfy the condition $-\lambda_{c}(\chi)<\lambda<\lambda_{c}(\chi)$. In particular (7) shows that there exist no ghost states.

We want to discuss some consequence of the above formulas. First of all, all parameters of our theory are fixed by describing the solution of our problem in $\mathscr{H}(1,1)$, there is no further arbitrariness for higher sectors. Since we have a renormalization of the internal energy, we will also write $H_{\chi}=H_{0}(U)+V(\chi)$ and treat $V(\chi)$ as a perturbation. $V(\chi)$ is still a bounded operator in each sector.

Secondly (6) shows that the operator

$$
V^{*}(P, \chi)=Z_{\chi}^{1 / 2} V^{*}(P)+\lambda \int \frac{\chi(\omega)}{U-\omega} N^{*}\left(\frac{m_{3}}{m_{1}} P+q\right) \Theta^{*}\left(\frac{m_{2}}{m_{1}} P-q\right) d^{3} q
$$

11 Commun. math. Phys., Vol. 10 
applied to the vacuum $\Omega$ gives the 1-particle $V$-state. Since the 1-particle states of $\Theta$ and $N$ are equal to the free 1-particle states, one has solved the 1-particle problem which is the first step in the Haag-Ruelle scattering theory [10]:

Theorem 1. Let $f\left(P_{1}, P_{2} \ldots P_{n_{1}}, k_{1} \ldots k_{n_{2}}, l_{1} \ldots l_{n_{3}}\right)$ be smooth and fast decreasing. Define

$$
\begin{aligned}
& f\left(P_{1}, \ldots, P_{n_{1}}, k_{1} \ldots k_{n_{2}}, l_{1} \ldots l_{n_{3}}, t\right)=f\left(P_{1} \ldots P_{n_{1}}, k_{1} \ldots k_{n_{2}}, l_{1} \ldots l_{n_{3}}\right) \\
& \cdot \exp \left\{-i \sum_{j_{1}=1}^{n_{1}}\left(U+\frac{P_{j_{1}}^{2}}{2 m_{1}}\right) t-i \sum_{j_{2}=1}^{n_{3}} \frac{k_{j_{2}}^{2}}{2 m_{2}} t-i \sum_{j_{3}=1}^{n_{3}} \frac{l_{j_{3}}^{2}}{2 m_{3}} t\right\} \\
& \left|V^{n_{1}} \Theta^{n_{2}} N^{n_{3}}(f, t, \chi)\right\rangle=\int \prod_{j_{1}=1}^{n_{1}} d^{3} P_{j_{1}} V^{*}\left(P_{j_{1}}, \chi\right) \int \prod_{j_{2}=1}^{n_{2}} d^{3} k_{j_{2}} \Theta^{*}\left(k_{j_{2}}\right) \\
& \cdot \int \prod_{j_{3}=1}^{n_{3}} d^{3} l_{j_{3}} N^{*}\left(l_{j_{3}}\right) f\left(P_{1} \ldots P_{n_{1}}, k_{1} \ldots k_{n_{2}}, l_{1} \ldots l_{n_{3}}, t\right) \Omega
\end{aligned}
$$

then the strong limit of

$$
\left(\exp i H_{\chi} t\right)\left|V^{n_{1}} \Theta^{n_{2}} N^{n_{3}}(f, t, \chi)\right\rangle
$$

for $t \rightarrow \pm \infty$ exists.

For the proof one takes the time derivative of (9) and shows that the norm of the expression so obtained is $0\left(|t|^{-3 / 2}\right)$ for large $|t|$. The theorem then follows from a standard argument used in the Haag-Ruelle scattering theory.

The third and most important consequence of the above relations is that (3), (5), (6) still make sense for $\chi \equiv 1$. The relations (7), (8) are then also valid. Therefore $r^{S}(\chi, z)$ and $u^{ \pm}(\chi)$ for $\chi \equiv \mathbf{1}$ describe the resolvent and the unitary scattering operators of local Hamiltonian $H^{S}$ in $\mathscr{H}_{2}$. Thus $H=H^{S}+(2 \mathscr{M})^{-1} \mathscr{P}^{2}$ is a local selfadjoint Hamiltonoperator in $\mathscr{H}(1,1)$. It may be shown that the domains of definition $H$ and $H_{0}(U)$ are different but have a nontrivial intersection:

Let $h^{ \pm}$be the hyperplane in $\mathscr{H}_{2}$ consisting of all $(g, f)$ such that

$$
g+\lambda \int \frac{f(q)}{H(\omega-U \mp i 0)} d^{3} q=0 .
$$

Choose $(g, f) \in h^{ \pm}$, where $f$ is smooth with compact support. Then

$$
(g, f) \in \mathscr{D}\left(H_{0}^{S}(U)\right) \cap \mathscr{D}\left(H^{S}\right) .
$$

In the next paragraph we will construct an operator $r(z)$ in each sector $\mathscr{H}\left(N_{1}, N_{2}\right)$ which will be the resolvent of a selfadjoint Hamiltonoperator $H . r(z)$ will be the limit $\chi \rightarrow 1$ of $r(\chi, z)$ (in a sense made precise below), where $r(\chi, z)$ is the resolvent of $H_{\chi}$.

In $\S 3$ we will discuss the scattering theory of $H$ in $\mathscr{H}(2,1)$ employing techniques which have been extensively used by FADDEEv [11]. 


\section{§ 2. Existence Proof for a Local Selfadjoint Hamiltonoperator $\boldsymbol{H}$}

We consider $H_{\chi}$ restricted to $\mathscr{H}\left(N_{1}, N_{2}\right)$. To begin with it will be convenient to construct a theory with distinguishable particles for the case with cutoff.

Def. 1. A configuration $\Gamma$ is a (possibly empty) set of ordered pairs $(i, j)$

$$
\begin{gathered}
1 \leqq i \leqq N_{1}, 1 \leqq j \leqq N_{2} \\
\Gamma=\left(i_{1}, j_{1}\right) \cup \cdots \cup\left(i_{k}, j_{k}\right) \quad 0 \leqq k \leqq \operatorname{Min}\left(N_{1}, N_{2}\right)
\end{gathered}
$$

with

We put

$$
i_{l} \neq i_{l^{\prime}}, j_{l} \neq j_{l^{\prime}} \quad \text { for } \quad l \neq l^{\prime} .
$$

$$
\begin{aligned}
\Gamma(1) & =\left\{i_{l}\right\}_{1 \leqq l \leqq k}, \Gamma(2)=\left\{j_{i}\right\}_{1 \leqq l \leqq k} \\
\Gamma(1)^{c} & =\left\{i \mid 1 \leqq i \leqq N_{1}, i \notin \Gamma(1)\right\} \\
\Gamma(2)^{c} & =\left\{j \mid 1 \leqq j \leqq N_{2}, j \notin \Gamma(2)\right\} \\
|\Gamma| & =k ; \quad\left|\Gamma(1)^{c}\right|=N_{1}-|\Gamma| ; \quad\left|\Gamma(2)^{c}\right|=N_{2}-|\Gamma| .
\end{aligned}
$$

Let $G$ be the set of all configurations. The Hilbert space $\mathscr{H}_{1}\left(N_{1}, N_{2}\right)$ is defined as follows:

An element $f$ in $\mathscr{H}_{1}\left(N_{1}, N_{2}\right)$ is a set of functions

$$
f=\left\{f_{\Gamma}\right\}_{\Gamma \in G}
$$

with $f_{T} \in \mathscr{L}^{2}\left(\mathbb{R}^{3\left(N_{1}+N_{2}-\mid \Gamma_{\mid}^{\prime}\right)}\right)$. The linear structure and the norm are given by

$$
\begin{aligned}
\lambda f+\mu g & =\left\{\lambda f_{\Gamma}+\mu g_{\Gamma}\right\}_{\Gamma \in G} \\
\|f\|^{2} & =\sum_{\Gamma \in G}\left\|f_{\Gamma}\right\|^{2} .
\end{aligned}
$$

The system of variables for $f_{\Gamma}$ is a system of 3-momenta

$\left\{P_{\left(i_{1}, j_{1}\right)} \ldots P_{\left(i \mid \Gamma_{i}, j_{|\Gamma|}\right)}, k_{i^{1}} \ldots k_{i}|\Gamma(1) c|, l_{j^{1}} \ldots l_{j|\Gamma(2) c|}\right\}=(P, k, l)_{\Gamma}$

$i^{l} \in \Gamma(1)^{c}, j^{l^{\prime}} \in \Gamma(2)^{c} ; 1 \leqq l \leqq\left|\Gamma(1)^{c}\right| ; 1 \leqq l^{\prime} \leqq\left|\Gamma(2)^{c}\right|$

$\left(i_{l^{\prime \prime}}, j_{l^{\prime \prime}}\right) \in \Gamma, 1 \leqq l^{\prime \prime} \leqq|\Gamma|$.

$f_{\Gamma}$ is to be interpreted as a wave function for the $V$-particles $V(i, j)$ $((i, j) \in \Gamma)$, the $\Theta$-particles $\Theta(i)\left(i \in \Gamma(1)^{c}\right)$ and the $N$-particles $N(j)$ $\left(j \in \Gamma(2)^{c}\right)$.

The free Hamiltonoperator is defined as

$$
\begin{aligned}
& \left(H_{01}\left(U_{0}\right) f\right)_{\Gamma}\left((P, k, l)_{\Gamma}\right) \\
& \quad=\left\{\sum_{(i, j) \in \Gamma}\left(U_{0}+\frac{P_{(i, j)}^{2}}{2 m_{1}}\right)+\sum_{i \in \Gamma^{\prime}(1) \mathrm{c}} \frac{k_{i}^{2}}{2 m_{2}}+\sum_{j \in \Gamma(2) \mathrm{c}} \frac{l_{j}^{2}}{2 m_{3}}\right\} f_{\Gamma}\left((P, k, l)_{\Gamma}\right) .
\end{aligned}
$$

If we put

$$
\begin{aligned}
\left(A^{(i, j)}(\chi) f\right)_{\Gamma} & =\delta U_{\chi} f_{\Gamma} \quad \text { if } \quad(i, j) \in \Gamma \\
& =0 \quad \text { otherwise }
\end{aligned}
$$


we get

$$
H_{01}\left(U_{0}\right)=H_{01}(U)+\sum_{(i, j)} A^{(i, j)}(\chi) .
$$

Now a linear operator $A$ in $\mathscr{H}_{1}\left(N_{1}, N_{2}\right)$ is given by its matrixelements $A_{\Gamma, \Gamma^{\prime}}$. In particular we want to construct a Hamiltonoperator $H_{1}(\chi)$. Consider the bounded operators $A^{(i, j)<}(\chi)$ and $A^{(i, j)>}(\chi)=\left(\mathrm{A}^{(i, j)<}(\chi)\right)^{\dagger}$ where $A^{(i . j)<}(\chi)$ is defined as follows

$$
A^{(i, j)<}(\chi)_{\Gamma, \Gamma^{\prime \prime}}=0 \text { if } \quad \Gamma \neq \Gamma^{\prime} \cup(i, j)
$$

and if $\Gamma=\Gamma^{\prime} \cup(i, j)$ we put

$$
\begin{aligned}
& \left(A^{(i, j)<}(\chi)_{\Gamma, \Gamma^{\prime}} f_{\Gamma^{\prime \prime}}\right)\left((P, k, l)_{\Gamma}\right) \\
& \quad=\lambda_{0} \int \chi(\omega(i, j)) f_{\Gamma^{\prime}}\left((P, k, l)_{\Gamma^{\prime}}\right) d^{3} q(i, j)
\end{aligned}
$$

where

$$
\begin{aligned}
2 \mu \omega(i, j) & =q^{2}(i, j) ; \quad m_{1} q(i, j)=m_{2} l_{j}-m_{3} k_{i} \\
P_{(i, j)} & =k_{i}+l_{j} \\
\left\{(P, k, l)_{\Gamma}, k_{i}, l_{j}\right\} & =\left\{(P, k, l)_{\Gamma^{\prime}}, P_{(i, j)}\right\} .
\end{aligned}
$$

$A^{(i, j)<}(\chi)$ is thus an operator which destroys the particles $\Theta(i)$ and $N(j)$ and creates $V(i, j)$.

The Hamiltonoperator of our system of distinguishable particles is now defined to be

$$
\left.\begin{array}{rl}
H_{1}(\chi) & =H_{01}(U)+\sum_{(i, j)}\left(A^{(i, j)<}(\chi)+A^{(i, j)>}(\chi)+A^{(i, j)}(\chi)\right. \\
& =H_{01}(U)+V_{1}(\chi) .
\end{array}\right\}
$$

$V_{1}(\chi)$ is bounded and selfadjoint, so by the theorem of KaTo [9] $H_{1}(\chi)$ is selfadjoint and the domains of definition of $H_{1}(\chi)$ and $H_{01}(U)$ coincide. Let $\gamma_{n}$ be the symmetric group of $n$ objects. In $\mathscr{H}_{1}\left(N_{1}, N_{2}\right)$ there is a (canonically defined) unitary representation $U$ of $\gamma_{N_{1}} \times \gamma_{N_{2}}$ which a commutes with $H_{1}(\chi)$. Let $\overline{\mathscr{H}}\left(N_{1}, N_{2}\right)$ be the closed linear subspace of $\mathscr{H}_{1}\left(N_{1}, N_{2}\right)$ consisting of all $f$ with

$$
\hat{U}\left(Q_{1}, Q_{2}\right) f=\left(\operatorname{sign} Q_{2}\right) \cdot f ; \quad\left(Q_{1}, Q_{2}\right) \in \gamma_{N_{1}} \times \gamma_{N_{2}} .
$$

Let $\bar{H}(\chi)$ be the restriction of $H_{1}(\chi)$ to $\overline{\mathscr{H}}\left(N_{1}, N_{2}\right)$. Then the theories $\left(H(\chi), \mathscr{H}\left(N_{1}, N_{2}\right)\right)$ and $\left(\bar{H}(\chi), \overline{\mathscr{H}}\left(N_{1}, N_{2}\right)\right)$ are unitarily equivalent.

We want to inspect the resolvent $r(\chi, z)$ of $H_{1}(\chi)$ :

Let $d(z)$ for complex $z$ be the distance of $z$ to the intervall $\left[\operatorname{Min}\left(N_{1}, N_{2}\right) U, \infty\right)$. Then the resolvent $r_{0}(z)$ of $H_{01}(U)$ satisfies the estimate

$$
\left\|r_{0}(z)\right\| \leqq C d^{-1}(z) .
$$

Since $V_{1}(\chi)$ is a bounded operator, (11) therefore implies the convergence of the Born series of $r(\chi, z)$ for sufficiently large $d(z)$ :

$$
r(\chi, z)=r_{0}(z) \sum_{n=0}^{n}\left(V_{1}(\chi) r_{0}(z)\right)^{n}
$$


In order to perform a partial summation in (12) we need some notations:

Def. 2. An index sequence $I(n)$ of length $n \geqq 1$ is an ordered sequence of symbols $a_{l}(1 \leqq l \leqq n)$

$$
I(n)=\left(a_{1} \ldots a_{n}\right)
$$

where each $a_{l}$ is of the form $(i, j)$ or $(i, j)<$ or $(i, j)>\left(1 \leqq i \leqq N_{1}\right.$, $\left.1 \leqq j \leqq N_{2}\right) . a_{l}$ is called the l-th value of $I(n)$. If $a_{l}$ is of the form $(i, j)$ we call $a_{l}$ a selfenergy term. $I(0)$ is defined to be the empty set.

The number of index sequences of length $n$ is $\left(3 N_{1} N_{2}\right)^{n}$. To each $I(n)$ and $z(d(z)>0)$ we associate a bounded linear operator:

$$
\begin{aligned}
& A(I(n), \chi, z)=r_{0}(z) \prod_{l=1}^{n}\left\{A^{a_{l}}(\chi) r_{0}(z)\right\} \quad n \geqq 1 \\
& A(I(0), \chi, z)=r_{0}(z) .
\end{aligned}
$$

For sufficiently large $d(z)$ we obtain

$$
r(\chi, z)=r_{0}(z)+\sum_{n=1}^{\infty} \sum_{I(n)} A(I(n), \chi, z) .
$$

Def. 3. $I(n)$ is said to contain a polarization of type $(i, j)$ if there exist $l_{1}, l_{2}\left(l_{1}<l_{2}\right)$ such that

$$
a_{l_{1}}=(i, j)<; \quad a_{l_{2}}=(i, j)>
$$

and for all $l^{\prime}\left(l_{1}<l^{\prime}<l_{2}\right) a_{l^{\prime}}$ is of the form

$$
a_{l^{\prime}}=\left(i^{\prime}, j^{\prime}\right)(\gtrless) \quad i^{\prime} \neq i ; j^{\prime} \neq j \text {. }
$$

Def. 4. $I(n)(n \geqq 1)$ is called polarized, if there exists an $m$ such that $I(n)$ contains $m$ polarizations and $n-2 m$ selfenergy terms. Such index sequences we denote by $I^{P}(n)$. By definition $I(0)$ is polarized.

Def. 5. Let $I(n)=\left(a_{1} \ldots a_{n}\right) n \geqq 1$

$$
I^{\iota, l^{\prime}}(n)=\left(a_{l} a_{l+1} \ldots a_{l^{\prime}-1} a_{l^{\prime}}\right) \quad l \leqq l^{\prime} .
$$

$I(n)$ is called renormalized if for all $\left(l, l^{\prime}\right) 1 \leqq l \leqq l^{\prime} \leqq u, I^{l, l^{\prime}}(n)$ is not polarized. In particular $I(n)$ contains no selfenergy terms. We will denote renormalized index sequences by $I^{R}(n) . I(0)$ is by definition renormalized. Let $I(n)$ be given with $A(I(n), \chi, z) \neq 0$. If $I^{l_{1}, l_{2}}(n)$ and $I^{l_{1}^{\prime}, l_{2}{ }^{\prime}}(n)$ are polarized and $l_{1} \leqq l_{1}^{\prime} \leqq l_{2}+1$ then also $I^{l_{1}, \max \left(l_{2}, l_{2}^{\prime}\right)}(n)$ is polarized. This shows the existence of a unique maximal set of nonintersecting intervals $\left[l_{1}{ }^{i}, l_{2}{ }^{i}\right] 1 \leqq i \leqq m(0 \leqq m \leqq n)$ such that each $I^{l_{1}{ }^{i}, l_{2}{ }^{i}}(n)$ is polarized and not properly contained in any polarized $I^{l, l^{\prime}}(n)$. Put

$$
I(p)=a_{i_{1}} \ldots a_{i_{p}} ; \quad 1 \leqq i_{k}<i_{k+1} \leqq n ; \quad a_{i_{k}} \in I(n) .
$$

$\left\{i_{k}\right\}_{1 \leqq k \leqq p}$ is defined to be the complement of the above intervals in $[1, n]$, therefore $p=n-\sum_{i=1}^{m}\left(l_{2}{ }^{i}-l_{1}{ }^{i}+1\right)$. It is easy to show that $I(p)$ 
is renormalized. This gives for large $d(z)$

where

$$
\begin{aligned}
r(\chi, z)= & \sum_{n=0}^{\infty} \sum_{I^{R}(n)} \sum_{l_{1}=0}^{\infty} \sum_{I^{P}\left(l_{1}\right)} \cdots \sum_{l_{n+1}=0}^{\infty} \sum_{I^{P}\left(l_{n+1}\right)} \prod_{k=1}^{n} \\
& \cdot\left\{A\left(I^{P}\left(l_{k}\right), \chi, z\right) A^{a_{k}}(\chi)\right\} A\left(I^{P}\left(l_{n+1}\right), \chi, z\right)
\end{aligned}
$$

Putting

$$
I^{R}(n)=\left(a_{1} \ldots a_{n}\right)
$$

and

$$
r_{0}^{R}(\chi, z)=\sum_{l=0}^{\infty} \sum_{I^{P}(l)} A\left(I^{P}(l), \chi, z\right)
$$

(13) gives

$$
B\left(I^{R}(n), \chi, z\right)=r_{0}^{R}(\chi, z) \prod_{k=1}^{n}\left\{A^{a_{k}}(\chi) r_{0}^{R}(\chi, z)\right\} .
$$

$$
r(\chi, z)=r_{0}^{R}(\chi, z)+\sum_{n=1}^{\infty} \sum_{I R(n)} B\left(I^{R}(n), \chi, z\right) .
$$

We call $r_{0}^{R}(\chi, z)$ the renormalized propagator and (15) the renormalized Born series. Our intention is to show that (15) still makes sense for $\chi \equiv 1$ and sufficiently large $d(z)$. To this end we have to inspect $r_{0}^{R}(\chi, z)$ more closely.

(11) and (14) immediately give

\section{Lemma 1.}

$$
\lim _{d(z) \rightarrow \infty}\left\|z r_{0}^{R}(\chi, z)-\mathbf{1}\right\|=0 .
$$

Since the full $l$-particle $V$-propagator is known [cf. (3)], $r_{0}^{R}(\chi, z)$ may also be obtained in a different way ([12]): Let $H_{0}(\chi, z)=z ; H_{1}(\chi, z)$ $=H(\chi, z)$ and define $H_{n+1}(\chi, z)$ inductively by

$$
\frac{1}{H_{n+1}(\chi, z)}=\frac{1}{H_{n}(\chi, z)}-\frac{1}{\pi} \int_{-U}^{\infty} \frac{1}{H_{n}(\chi, z-\tau)} \operatorname{Im} \frac{1}{H_{1}(\chi, \tau)} d \tau .
$$

$\operatorname{Im} H_{1}^{-1}(\chi, \tau)$ is integrable in $[-U, \infty)$ so complete induction immediately gives

Lemma 2.

a) $H_{n}^{-1}(\chi, z)$ is analytic in the cut plane $-U \leqq z<\infty$ except for a simple pole at $z=0$. The residue of this pole is 1 .

b) $H_{n}^{-1}\left(\chi, z^{*}\right)^{*}=H_{n}^{-1}(\chi, z) ; \lim _{d(z) \rightarrow \infty} z H_{n}^{-1}(\chi, z)=Z_{\chi}^{-n}$,

c) $H_{n}{ }^{-1}\left(\chi, z-p^{2}\right)$ is in $\mathscr{L}^{v}\left(\mathbb{R}^{3}\right) ; \nu=2, \infty$.

For the corresponding norms we get

$$
\begin{gathered}
\left\|Z_{\chi}{ }^{n} H_{n}^{-1}\left(\chi, z-{ }^{2}\right)\right\|_{2} \leqq C_{2}(n, \varepsilon) d(z)^{-1 / 4+\varepsilon} \quad 0<\varepsilon<\frac{1}{4}, \\
\left\|Z_{\chi}^{n} H_{n}^{-1}\left(\chi, z-{ }^{-2}\right)\right\|_{\infty} \leqq C_{\infty}(n) d(z)^{-1} .
\end{gathered}
$$




$$
\begin{aligned}
& r_{0}^{R}(\chi, z) \text { is then given by } \\
& \qquad\left(r_{0}^{R}(\chi, z) f\right)_{\Gamma}\left((P, k, l)_{\Gamma}\right)=\frac{Z_{\chi}|\Gamma| f_{\Gamma}\left((P, k, l)_{\Gamma}\right)}{H_{|\Gamma|}\left(\chi, z-E\left((P, k, l)_{\Gamma}\right)\right)} \\
& E\left((P, k, l)_{\Gamma}\right)=\sum_{(i, j) \in \Gamma}\left(\frac{P_{(i, j)}^{2}}{2 m_{1}}+U\right)+\sum_{i \in \Gamma(1) \circ} \frac{k_{i}^{2}}{2 m_{2}}+\sum_{j \in \Gamma(2)^{\circ}} \frac{l_{j}^{2}}{2 m_{3}} .
\end{aligned}
$$

The important point is now that Lemma 2 still holds for $\chi \equiv 1$. In particular $C_{2}(n, \varepsilon)$ and $C_{\infty}(n)$ may be chosen independently of $\chi$. (17) then defines for $\chi \equiv 1$ a bounded operator $r_{0}^{R}(z)$ which is analytic in $z$ for $d(z)>0$.

Remark 1. Lemma 1 still holds for $r_{0}^{R}(z)$. Let

$$
\begin{aligned}
C_{2}(\varepsilon)= & \operatorname{Max} C_{2}(n, \varepsilon) ; \\
& 0 \leqq n \leqq \operatorname{Min}\left(N_{1}, N_{2}\right) \\
C_{\infty}= & \operatorname{Max} C_{\infty}(n) . \\
& 0 \leqq n \leqq \operatorname{Min}\left(N_{1}, N_{2}\right)
\end{aligned}
$$

Then Lemma 2 gives ( $\chi \equiv 1$ included):

Lemma 3. For $d(z) \neq 0 r_{0}^{R}(\chi, z)$ is an analytic operatorvalued function with

$$
\left\|r_{0}^{R}(\chi, z)\right\| \leqq C_{\infty} d(z)^{-1} .
$$

Let $p$ be any of the momenta in $(P, k, l)_{\Gamma}$. Then $\left(r_{0} R(\chi, z) f\right)_{\Gamma}$ may be regarded as a $\mathscr{L}^{1}$ function in $p$ and as a $\mathscr{L}^{2}$ function in the remaining variables. If we denote the so defined norm by \|\|$_{\Gamma}$ we obtain

$$
\left\|\left(r_{0}^{R}(\chi, z) f\right)_{\Gamma}\right\|_{\Gamma} \leqq\left\|f_{r^{\prime}}\right\| C_{2}(\varepsilon) d(z)^{-\frac{1}{4}+\varepsilon} .
$$

Remark 2. $p$ may also be chosen to be one of the momenta one obtains on performing a linear transformation on $(P, k, l)_{\Gamma}$. Also \|\|$_{\Gamma}$ evidently depends on the chosen $p$. Since in future, however, we shall only have to deal with a finite number of different momentum systems for each $\Gamma$, $C_{2}(\varepsilon)$ may be chosen independently of all such $p$ and all $\Gamma$. We also put

$$
\|f\|_{\sim}=\sup _{\Gamma \in G}\left\|f_{\Gamma}\right\|_{\Gamma}
$$

Before we can formulate the next theorem, we need one more definition:

Def. 6. The final state configuration $\Gamma\left(I^{R}(n)\right)$ of $I^{R}(n)$ is defined to be the maximal configuration

$$
\Gamma\left(I^{R}(n)\right)=\left(\bar{i}_{1}, \bar{j}_{1}\right) \cup \cdots\left(\bar{i}_{k}, \bar{j}_{k}\right) ; \quad 0 \leqq k \leqq \operatorname{Min}\left(n, \operatorname{Min}\left(N_{1}, N_{2}\right)\right)
$$

such that the following statements hold.

For each $m(1 \leqq m \leqq k)$ there exists an $l(m) 1 \leqq l(m) \leqq n$ such that $a_{l(m)}$ is of the form $\left(\bar{i}_{m}, \bar{j}_{m}\right)>$ and $a_{l}$ is of the form $(i, j) \longleftrightarrow i \neq \bar{i}_{m}, j \neq \bar{j}_{m}$ for all $l 1 \leqq l<l(m)$. Maximal means that $\mid \Gamma\left(I^{R}(n) \mid\right.$ shall be maximal. 
Thus $\Gamma\left(I^{R}(n)\right)$ is unique. Also the numeration is to be chosen in such a way that $l(m)<l\left(m^{\prime}\right)$ for $m<m^{\prime}$.

By Def. $6\left(B\left(I^{R}(n), \chi, z\right) f\right)_{\Gamma}$ is zero unless $\Gamma \cup \Gamma\left(I^{R}(n)\right)$ is a configuration. Applying a linear transformation (out of a fixed finite set of linear transformations) on $(P, k, l)_{\Gamma}$, we may therefore obtain a system $(p)_{\Gamma}^{\Gamma\left(I^{R}(n)\right)}$ of momenta, which contains the variables

$$
q\left(\bar{i}_{m}, \bar{j}_{m}\right)=m_{1}^{-1}\left(m_{2} l_{\bar{j}_{m}}-m_{3} k_{\bar{j}_{m}}\right) \quad\left(\bar{i}_{m}, \bar{j}_{m}\right) \in \Gamma\left(I^{R}(n)\right) .
$$

Let now $A^{(i, j)<}$ and $A^{(i, j)>}$ be the formal operators we obtain setting $\chi \equiv 1$ in $A^{(i, j)<}(\chi)$ and $A^{(i, j)>}(\chi)$. Define $B\left(I^{R}(n), z\right)$ in the same way. We will prove

Theorem 2. For $d(z)>0 B\left(I^{R}(n), z\right)$ is a bounded linear operator in $\mathscr{H}_{1}\left(N_{1}, N_{2}\right)$ which is analytic in $z$.

The norm satisfies the estimate

$$
\left\|B\left(I^{R}(n), z\right)\right\| \leqq \lambda_{0}^{n} C_{\infty} C_{2}^{n}(\varepsilon) d(z)-\frac{n}{4}-1+n \varepsilon .
$$

$B\left(I^{R}(n), z\right)_{\Gamma, \Gamma^{\prime}}$ is equal to zero unless $\Gamma \cup \Gamma\left(I^{R}(n)\right)$ is a configuration. If $\left.p \in(p)_{\Gamma} \Gamma^{R}(n)\right)$ is none of the $q\left(\bar{i}_{m}, \bar{j}_{m}\right), \quad\left(\bar{i}_{m}, \bar{j}_{m}\right) \in \Gamma\left(I^{R}(n)\right)$ then $\left(B\left(I^{R}(n), z\right) f\right)^{R}$ is a $\mathscr{L}^{1}$ function in $p$ and a $\mathscr{L}^{2}$ function in the remaining variables. The norm \|\|$_{\sim}$ in these variables satisfies

$$
\left\|B\left(I^{R}(n), z\right) f\right\|_{\sim} \leqq \lambda_{0}^{n}\|f\| C_{2}(\varepsilon)^{n+1} d(z)^{-\frac{n+1}{4}+(n+1) \varepsilon} .
$$

The proof proceeds by complete induction: $n=0$ is simply Lemma 3 .

Let now $n \geqq 1$ and consider $B\left(I^{R}(n), z\right)$. Define $l_{0}$ to be the smallest $l$, such that $a_{l}$ does not belong to any polarization. Since $I^{R}(n)$ is renormalized such a $l_{0}$ exists with $1 \leqq l_{0} \leqq \frac{n+1}{2} . I^{R}(n)$ then necessarily has the form

$$
\begin{gathered}
I^{R}(n)=\left(i_{1}, j_{1}\right)< \\
\cup\left(i_{2}, j_{2}\right)<\cup \cdots \cup\left(i_{l_{0}-1}, j_{l_{0}-1}\right)< \\
\cup\left(i_{l_{0}}, j_{l_{0}}\right)(<) \cup I^{l_{0}+1, n}(n)
\end{gathered}
$$

where $I^{l_{0}+1, n}(n)$ also is renormalized. So we may assume that theorem 2 already holds for $B\left(I^{l_{0}+1, n}(n), z\right)$. We consider the following two cases separately:

a) $a_{l_{0}}$ has the form $\left(i_{l_{0}}, j_{l_{0}}\right)<$. Then

and

$$
\left(i_{l_{0}}, j_{l_{0}}\right) \notin \Gamma\left(I^{l_{0}+1, n}(n)\right)
$$

$$
\left(i_{1}, j_{1}\right) \cup\left(i_{2}, j_{2}\right) \cdots \cup\left(i_{l_{0}-1}, j_{l_{0}-1}\right) \subset \Gamma\left(I^{l_{0}+1, n}(n)\right) .
$$

b) $a_{l_{0}}$ has the form $\left(i_{l_{0}}, j_{l_{0}}\right)>$. Then

and

$$
\left(i_{l_{0}}, j_{l_{0}}\right) \text { is the first term in } \Gamma\left(I^{R}(n)\right)
$$

$$
\left(i_{l_{0}}, j_{l_{0}}\right) \cup \Gamma\left(I^{l_{0}+1, n}(n)\right)=\left(i_{1}, j_{1}\right) \cup \cdots\left(i_{l_{0}-1}, j_{l_{0}-1}\right) \cup \Gamma\left(I^{R}(n)\right) .
$$


For the case a) we choose the system $(p)_{\Gamma}^{\Gamma\left(I^{l_{0}}+1, n_{(n)}\right)}$ to contain the momenta $q\left(i_{l}, j_{l}\right) 1 \leqq l \leqq l_{0}$.

By assumption $\left(B\left(I^{l_{0}+1, n}(n), z\right) f\right)_{\Gamma}$ is in $\mathscr{L}^{1}$ with respect to $q\left(i_{l_{0}}, j_{l_{0}}\right)$ and in $\mathscr{L}^{2}$ with respect to the remaining variables. Application of $A^{\left(i_{0}{ }_{0}, j_{l_{0}}\right)<}$ means we have to carry out the $q\left(i_{l_{0}}, j_{l_{0}}\right)$ integration and multiply by $\lambda_{0}$. Further application of $r_{0}^{R}(z)$ on $\left(A^{\left(i_{l_{0}-1}, \dot{j}_{0}-1\right)}<\right.$ $\left.B\left(l_{0}+1, n(n), z\right) f\right)_{\Gamma}$ gives a $\mathscr{L}^{1}$-function in $q\left(i_{l_{0}-1}, j_{l_{0}-1}\right)$ :

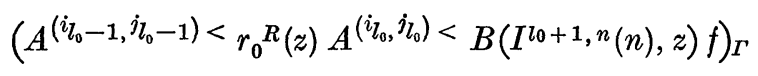

is therefore in $\mathscr{L}^{2}$ with respect to each variable etc., so finally

$$
\left(\prod_{l=1}^{l_{0}-1}\left\{A^{\left(i_{l}, j_{l}\right)<} r_{0}^{R}(z)\right\} A^{\left(i_{l_{0}}, j_{l_{0}}\right)<} B\left(I^{l_{0}+1, n}(n), z\right) f\right)_{\Gamma}
$$

is in $\mathscr{L}^{2}$ in each variable. A last application of $r_{0}^{R}(z)$ implies that $\left(B\left(I^{R}(n), z\right) f\right)_{\Gamma}$ even is in $\mathscr{L}^{1}$ for one arbitrary variable. We immediately obtain the estimates

$$
\begin{aligned}
& \left\|B\left(I^{R}(n), z\right) f\right\| \leqq \lambda_{0}^{l_{0}} C_{\infty} C_{2}^{l_{0}-1}(\varepsilon) d(z)^{-\frac{\left(l_{0}-1\right)}{4}-1+\left(l_{0}-1\right) \varepsilon}\left\|B\left(I^{l_{0}+1, n}(n), z\right) f\right\|_{\sim} \\
& \left\|B\left(I^{R}(n), z\right) f\right\|_{\sim} \leqq \lambda_{0}{ }^{l_{0}}\left(_{2}^{l_{0}}(\varepsilon) d(z)^{-\frac{l_{0}}{4}+l_{0} \varepsilon}\left\|B\left(I^{l_{0}+1, n}(n), z\right) f\right\|_{\sim} \cdot\right.
\end{aligned}
$$

The statement for $n$ then follows if we use the estimates for $B\left(I^{l_{0}+1, n}(n), z\right)$.

We turn to the case b):

If we apply $A^{\left(i_{l_{0}}, j_{l_{0}}\right)}>$ to $B\left(I^{l_{0}+1, n}(n), z\right) f$ then $\left(A^{i_{l_{0}}, j_{l_{0}}>} B\left(I^{l_{0}+1, n}(n), z\right) f\right)_{\Gamma}$ will be in $\mathscr{L}^{\infty}$ with respect to $q\left(i_{l_{0}}, j_{l_{0}}\right)$, in $\mathscr{L}^{2}$ for every remaining variable and even in $\mathscr{L}^{1}$ for one $p \in(p)_{\Gamma}^{\Gamma\left(I^{l_{0}}+1, n(n)\right)}$ which is not a $q(\bar{i}, \bar{j}),(\bar{i}, \bar{j}) \in \Gamma\left(I^{l_{0}+1, n}(n)\right)$. Application of $r_{0}{ }^{R}(z)$ gives a $\mathscr{L}^{1}$ function in $q\left(i_{l_{0}-1}, j_{l_{0}-1}\right)$. Therefore we may apply $A^{\left(i_{l_{0}-1}, j_{l_{0}-1}\right)<}$ etc., so finally

$$
\left(\prod_{l=1}^{l_{0}-1}\left(A^{\left(i_{l}, j_{l}\right)<} r_{0}^{R}(z)\right) A^{\left(i_{l_{0}}, j_{0}\right)>} B\left(I^{l_{0}+1, n}(n), z\right) f\right)_{\Gamma}
$$

will be in $\mathscr{L}^{\infty}$ with respect to $q\left(i_{l_{0}}, j_{l_{0}}\right)$, in $\mathscr{L}^{2}$ for every remaining variable and even in $\mathscr{L}^{1}$ for one $p \in(p)_{\Gamma}^{\Gamma\left(I^{R}(n)\right)}$ which is none of the momenta $q(\bar{i}, \bar{j}),(\bar{i}, \bar{j}) \in \Gamma\left(I^{R}(n)\right)$. A last application of $r_{0}{ }^{R}(z)$ gives a $\mathscr{L}^{2}$ function in $q\left(i_{l_{0}}, j_{l_{0}}\right)$, leaving the properties in the other variables unchanged. This gives the estimates

$$
\begin{aligned}
& \left\|B\left(I^{R}(n), z\right) f\right\| \leqq \lambda_{0}^{l_{0}} C_{2}^{l_{0}}(\varepsilon) d(z)^{-\frac{l_{0}}{4}+l_{0} \varepsilon}\left\|B\left(I^{l_{0}+1, n}(n), z\right) f\right\| \\
& \left\|B\left(I^{R}(n), z\right) f\right\|_{\sim} \leqq \lambda_{0}^{l_{0}} C_{2}^{l_{0}}(\varepsilon) d(z)^{-\frac{l_{0}}{4}+l_{0} \varepsilon}\left\|B\left(I^{l_{0}+1, n}(n), z\right) f\right\|_{\sim} .
\end{aligned}
$$

Using the estimates for $B\left(I^{l_{0}+1, n}(n), z\right)$ we again obtain the statement for $n$. Theorem 2 is proved. Theorem 2 and Lemma 1 (cf. remark 1) give 
Corollary 1. The series

$$
r(z)=\sum_{n=0}^{\infty} \sum_{I^{R}(n)} B\left(I^{R}(n), z\right)
$$

converges for sufficiently large $d(z)$ and we have

$$
\lim _{d(z) \rightarrow \infty}\|z r(z)-1\|=0 \text {. }
$$

Furthermore we have

Corollary 2. For sufficiently large $d(z)$

$$
r(z)=r\left(z^{*}\right)^{\dagger}
$$

and $r(z)$ commutes with the representation $\hat{\theta}$ of $\gamma_{N_{1}} \times \gamma_{N_{2}}$.

Proof. Since $d(z)=d\left(z^{*}\right)$ we may suppose $r\left(z^{*}\right)$ to be defined whenever $r(z)$ is defined. The first statement follows from

$$
r_{0}^{R}\left(z^{*}\right)^{\dagger}=r_{0}^{R}(z)
$$

and the following consideration: Let

and put

$$
I^{R}(n)=\left(a_{1} \ldots a_{n}\right)
$$

where

$$
\bar{I}^{R}(n)=\bar{a}_{n} \ldots \bar{a}_{2} \bar{a}_{1}
$$

$$
\begin{aligned}
\bar{a} & =(i, j)<\text { if } \quad a=(i, j)> \\
& =(i, j)>\text { if } \quad a=(i, j)<.
\end{aligned}
$$

Then the $\operatorname{map} I^{R}(n) \rightarrow \bar{I}^{R}(n)$ is a one to one map on the set of all renormalized index sequences of length $n$ such that $B\left(I^{R}(n), z\right)^{\dagger}$ $=B\left(\bar{I}^{R}(n), z^{*}\right)$. The second statement follows from a similar consideration: $\gamma_{N_{1}} \times \gamma_{N_{2}}$ acts in a canonical way as a transformation group on the set of all renormalized index sequences of length $n$ and therefore

$$
\hat{U}\left(Q_{1}, Q_{2}\right) \sum_{I^{R}(n)} B\left(I^{R}(n), z\right) t^{Y-1}\left(Q_{1}, Q_{2}\right)=\sum_{I^{R}(n)} B\left(I^{R}(n), z\right)
$$

for all $n,\left(Q_{1}, Q_{2}\right) \in \gamma_{N_{1}} \times \gamma_{N_{2}}$ and $z(d(z)>0)$, since $r_{0}^{R}(z)$ commutes with 0 .

For the next theorem we need a further lemma. Let $\chi_{N}$ be chosen in such a way that $0 \leqq \chi_{N} \leqq 1$ and $\chi_{N}(\omega)=1$ for $\omega \leqq N$.

Lemma 4. For $0 \leqq n \leqq \operatorname{Min}\left(N_{1}, N_{2}\right)$ we have

$$
\begin{aligned}
& \left\|Z_{\chi_{N}}{ }^{n} H_{n}{ }^{-1}\left(\chi_{N}, z-{ }^{2}\right)-Z_{1}{ }^{n} H_{n}{ }^{-1}\left(z-{ }^{2}\right)\right\|_{2} \leqq C_{2}(N, \varepsilon) d(z)^{-1 / 4+\varepsilon}, \\
& \left\|Z_{\chi_{N}}{ }^{n} H_{n}{ }^{-1}\left(\chi_{N}, z-{ }^{2}\right)-Z_{1}{ }^{n} H_{n}{ }^{-1}\left(z-{ }^{2}\right)\right\|_{\infty} \leqq C_{\infty}(N) d(z)^{-1} .
\end{aligned}
$$

Here $H_{n}(z)=H_{n}(\chi \equiv 1, z) ; Z_{1}=Z_{\chi=1}$, and $C_{2}(N, \varepsilon) \rightarrow 0, C_{\infty}(N) \rightarrow 0$ for $N \rightarrow \infty$ and fixed $\varepsilon$. 
Theorem 3. Let $G_{\tau}=\{z \mid d(z) \geqq \tau\}$.

Then for sufficiently large $\tau$

uniformly in $G_{\tau}$.

$$
\lim _{N \rightarrow \infty}\left\|r\left(\chi_{N}, z\right)-r(z)\right\|=0
$$

Proof. We want to give an estimate for

$$
\begin{gathered}
B\left(I^{R}(n), \chi_{N}, z\right)-B\left(I^{R}(n), z\right)=\sum_{k=0}^{n} H^{k}\left(I^{R}(n), \chi_{N}, z\right) \\
+\sum_{k=1}^{n} G^{k}\left(I^{R}(n), \chi_{N}, z\right) \\
H^{k}\left(I^{R}(n), \chi_{N}, z\right)=\prod_{l=1}^{k}\left(r_{0}^{R}\left(\chi_{N}, z\right) A^{a_{l}}\left(\chi_{N}\right)\right)\left(r_{0}^{R}\left(\chi_{N}, z\right)-r_{0}^{R}(z)\right) \\
\cdot \prod_{l=k+1}^{n}\left(A^{a_{l}} r_{0}{ }^{R}(z)\right) \\
G^{k}\left(I^{R}(n), \chi_{N}, z\right)=r_{0}^{R}\left(\chi_{N}, z\right) \prod_{l=1}^{k-1}\left(A^{a_{l}}\left(\chi_{N}\right) r_{0}^{R}\left(\chi_{N}, z\right)\right) \\
\cdot\left(A^{a_{k}}\left(\chi_{N}\right)-A^{a_{k}}\right) \prod_{l=k+1}^{n}\left(r_{0}^{R}(z) A^{l}\right) r_{0}^{R}(z) .
\end{gathered}
$$

First of all we remark that the estimates of theorem 2 also hold uniformly in $\chi_{N}$ for $B\left(I^{R}(n), \chi_{N}, z\right)$. According to our convention $\lambda_{0}\left(\chi_{N}\right)=Z_{\chi_{N}}{ }^{-1 / 2} \lambda$; $\lambda_{0}=Z_{1}{ }^{-1 / 2} \lambda$ with $\lambda$ fixed. Thus $\lambda_{0}\left(\chi_{N}\right) \leqq \lambda_{0}$. Remembering how we estimated each factor $r_{0}{ }^{R}(z)$ in $B\left(I^{R}(n), z\right)$, Lemma 4 immediately gives

$$
\begin{gathered}
\left\|H^{k}\left(I^{R}(n), \chi_{N}, z\right)\right\| \leqq \lambda_{0}{ }^{n} C^{n}(\varepsilon)\left[C_{2}(N, \varepsilon)+C_{\infty}(N)\right] d(z)-\frac{n+1}{4}+(n+1) \varepsilon \\
C(\varepsilon)=\operatorname{Max}\left(C_{2}(\varepsilon), C_{\infty}\right), \quad d(z) \geqq 1 .
\end{gathered}
$$

In order to estimate $G^{k}\left(I^{R}(n), \chi_{N}, z\right)$ we may assume that $a_{k}$ has the form $\left(i_{k}, j_{k}\right)<$. Otherwise we consider $\left(G_{k}\left(I^{R}(n), \chi_{N}, z\right)\right)^{\dagger}$. Now $A^{\left(i_{k}, j_{k}\right)<}$ corresponds to an integration over $q\left(i_{k}, j_{k}\right)$. All integrations of this kind have been estimated in theorem 2. The operator $A^{\left(i_{k}, j_{k}\right)<}\left(\chi_{N}\right)-A^{\left(i_{k}, j_{k}\right)<}$ corresponds to multiplication by $\lambda_{0}\left(\chi_{N}\right) \chi\left(\omega\left(i_{k}, j_{k}\right)\right)-\lambda_{0}$ and then integration over $q\left(i_{k}, j_{k}\right)$. Repeating the estimates of theorem 2 gives

$$
\begin{aligned}
\left\|G^{k}\left(I^{R}(n), \chi_{N}, z\right)\right\| & \leqq \lambda_{0}{ }^{n} C^{n}(\varepsilon) C^{\prime}(N, \varepsilon) d(z)^{-\frac{n+1}{4}+(n+1) \varepsilon} \\
d(z) & \geqq 1
\end{aligned}
$$

where $C^{\prime}(N, \varepsilon) \rightarrow 0$ for $N \rightarrow \infty$ and $\varepsilon$ fixed. $C^{\prime}(N, \varepsilon)$ is of course independent of $k, I^{R}(n)$ and $z$. If we sum the estimates (22) and (23) theorem 3 follows.

Corollary 1. $r(z)$ is a pseudoresolvent in $G_{\tau}$ for sufficiently large $\tau$. Proof. Consider the identity

$$
r\left(\chi_{N}, z_{1}\right)-r\left(\chi_{N}, z_{2}\right)=\left(z_{2}-z_{1}\right) r\left(\chi_{N}, z_{1}\right) r\left(\chi_{N}, z_{2}\right)
$$

which is valid because $r\left(\chi_{N}, z\right)$ is the resolvent of $H_{1}\left(\chi_{N}\right)$. 
According to theorem 3 we may take the limit $N \rightarrow \infty$ for sufficiently large $\tau$ and obtain

$$
r\left(z_{1}\right)-r\left(z_{2}\right)=\left(z_{2}-z_{1}\right) r\left(z_{1}\right) r\left(z_{2}\right) .
$$

We are now ready to prove the existence of a local Hamiltonian: Choose $\tau_{0}$ such that $r(z)$ exists for $z \in G_{\tau_{0}}$ and (21), (24) hold. Let $N(z)$ be the kernel of $r(z)\left(z \in G_{\tau_{0}}\right)$. Because of (24) $N(z)$ does not depend on $z$, therefore (20) implies that $N(z)$ is zero.

Lemma 5 [13]. A pseudoresolvent is a resolvent of a closed linear operator iff its kernel is zero.

Thus we may define

$$
H_{1}=z-r(z)^{-1} \text {. }
$$

Because of (24) $H_{1}$ is independent of $z \in G_{\tau_{0}}$. Since $N(z)$ is zero, (21) shows that the range of $r(z)$ is dense: $H_{1}$ is densely defined.

Lemma 6 ([14], Chap. XII). Let $T$ be a densely defined linear operator in a Hilbert space such that $T^{-1}$ exists and is densely defined. Then $\left(T^{\dagger}\right)^{-1}$ exists and is equal to $\left(T^{-1}\right)^{\dagger}$.

If we apply this lemma to $r(z)$ and use (21), (25) gives

Theorem 4. $H_{1}$ is a selfadjoint linear operator. The spectrum of $H_{1}$ is bounded below.

The last statement follows from the fact that $G_{\tau_{0}}$ contains the intervall

$$
\left(-\infty,-\tau_{0}+\operatorname{Min}\left(N_{1}, N_{2}\right) U\right] \text {. }
$$

Because of corollary 2 of theorem 2 the same arguments give a selfadjoint operator $H$ in $\overline{\mathscr{H}}\left(N_{1}, N_{2}\right)$ which is the restriction of $H_{1}$ to $\overline{\mathscr{H}}\left(N_{1}, N_{2}\right)$. Theorem 3 permits us to interpret $H$ as the local Hamiltonoperator of the Galilean invariant Lee model.

\section{$\S 3$. Scattering Theory in the $V \Theta-N \Theta \Theta-S e c t o r$}

In this sector we want to discuss $H$ in the sector $\mathscr{H}(2,1)$. The discussion in $\mathscr{H}(1,2)$ proceeds in the same way. Since $\mathscr{H}(2,1)$ also describes 3 particle configuration, namely one $N$ particle with momentum $l$ and two $\Theta$ particles with momenta $k_{1}, k_{2}$, it will be useful to introduce the following variables

$$
\begin{aligned}
P= & k_{1}+k_{2}+l, \\
q_{1}= & m_{1}^{-1}\left(m_{2} l_{2}-m_{3} k_{1}\right) ; \quad q_{2}=m_{1}^{-1}\left(m_{2} l-m_{3} k_{2}\right) \\
p_{1}= & \left(m_{1}+m_{2}\right)^{-1}\left(m_{2}\left(l+k_{1}\right)-m_{1} k_{1}\right) ; \\
p_{2}= & \left(m_{1}+m_{2}\right)^{-1}\left(m_{2}\left(l+k_{2}\right)-m_{1} k_{2}\right) \\
& 2 \mu \omega_{1}=q_{1}^{2} \quad 2 \mu \omega_{2}=q_{2}{ }^{2} .
\end{aligned}
$$


The following relations hold

$$
\begin{aligned}
q_{1} & =p_{2}+\frac{m_{2}}{m_{1}} p_{1} ; \quad q_{2}=p_{1}+\frac{m_{2}}{m_{1}} p_{2}, \\
\frac{l^{2}}{2 m_{3}}+\frac{k_{1}{ }^{2}}{2 m_{2}}+\frac{k_{2}{ }^{2}}{2 m_{3}}= & =\frac{P^{2}}{2\left(m_{1}+m_{2}\right)}+\frac{p_{1}^{2}}{2 \nu}+\omega_{1}=\frac{P^{2}}{2\left(m_{1}+m_{2}\right)}+\frac{p_{2}{ }^{2}}{2 v}+\omega_{2}, \\
v\left(m_{1}+m_{2}\right) & =m_{1} m_{2} .
\end{aligned}
$$

Since $\Theta$ is defined to be a boson, all expressions must be symmetric in $k_{1}$ and $k_{2}$, i. e. they must be invariant with respect to the substitution $\left(p_{1}, q_{1}\right) \leftrightarrow\left(p_{2}, q_{2}\right)$. We will often therefore simply write $(p, q)$. We may also identify $\mathscr{H}(2,1)$ with $\mathscr{H}_{1} \otimes \mathscr{H}_{2}$ where again $\mathscr{H}_{1}$ is the Hilbert space of the center of mass motion and $\mathscr{H}_{2}=\mathscr{L}^{2}\left(\mathbb{R}^{3}\right) \oplus \mathscr{L}^{2 s}\left(\mathbb{R}^{6}\right)$. Here $\mathscr{L}^{2 s}\left(\mathbb{R}^{6}\right)$ consists of all $f \in \mathscr{L}^{2}\left(\mathbb{R}^{6}\right)$ such that $f\left(p_{1}, q_{1}\right)=f\left(p_{2}, q_{2}\right)$. Since $H$ commutes with $(2 \mathscr{M})^{-1} \mathscr{P}^{2}$ it will suffice to consider $H^{S}$ $=H-(2 \mathscr{M})^{-1} \mathscr{P}^{2} . H^{S}$ only acts on $\mathscr{H}_{2}$. The resolvent $r^{S}(z)$ of $H^{S}$ then may be written as a $2 \times 2$ matrix with the kernel:

$$
r^{S}(z)\left(\tilde{p}, p, q ; \tilde{p}^{\prime}, p^{\prime}, q^{\prime}\right)=\left(\begin{array}{ll}
r_{11}^{S}(z)\left(\tilde{p}, \tilde{p}^{\prime}\right) & r_{12}^{S}(z)\left(\tilde{p} ; p^{\prime}, q^{\prime}\right) \\
r_{21}^{S}(z)\left(p, q ; \tilde{p}^{\prime}\right) & r_{22}^{S}(z)\left(p, q ; p^{\prime}, q^{\prime}\right)
\end{array}\right) .
$$

In analogy to $\S 1$ we further introduce $e^{\prime}$ and $r_{0}{ }^{R, S}(z)$ by

$$
\begin{aligned}
e^{\prime}\left(\tilde{p}, p, q ; \tilde{p}^{\prime}, p^{\prime}, q^{\prime}\right) & =\left(\begin{array}{cc}
Z_{1}^{1 / 2} \delta^{3}\left(\tilde{p}-\tilde{p}^{\prime}\right) & 0 \\
0 & \delta^{3}\left(p_{1}-p_{1}^{\prime}\right) \delta^{3}\left(q_{1}-q_{1}^{\prime}\right)
\end{array}\right), \\
r_{0}{ }^{R, S}(z)\left(\tilde{p}, p, q ; \tilde{p}^{\prime}, p^{\prime}, q^{\prime}\right) & =\left(\begin{array}{cc}
\frac{Z_{1}^{1 / 2} \delta^{3}\left(\tilde{p}-\tilde{p}^{\prime}\right)}{H\left(z-U-\frac{\tilde{p}^{\prime 2}}{2 v}\right)} & 0 \\
0 & \frac{\delta^{3}\left(p_{1}-p_{1}{ }^{\prime}\right) \delta^{3}\left(q_{1}-q_{1}{ }^{\prime}\right)}{z-\frac{p^{2}}{2 \nu}-\frac{q^{2}}{2 \mu}}
\end{array}\right) \\
\text { Putting } \quad r^{S}(z) & =\left(\begin{array}{c}
\left.e^{\prime}+r_{0}{ }^{R, S}(z) t(z)\right) r_{0}{ }^{R, S}(z)
\end{array}\right)
\end{aligned}
$$

then for sufficiently large $d(z)$ the renormalized Born Series for $r(z)$ gives the following expression for $t(z)$ :

where

$$
t_{11}(z)\left(\cdot, \tilde{p}^{\prime}\right)=\sum_{n=0}^{\infty} K(z)^{n} I(z)\left(\cdot, \tilde{p}^{\prime}\right)
$$

$$
\begin{array}{r}
I(z)\left(\tilde{p}, \tilde{p}^{\prime}\right)=\lambda^{2}\left(z-\frac{\tilde{p}^{\prime 2}}{2 v}-\left(\tilde{p}+\frac{m_{2}}{m_{1}} \tilde{p}^{\prime}\right)^{2} \frac{1}{2 \mu}\right)^{-1}=I(z)\left(\tilde{p}^{\prime}, \tilde{p}\right) \\
K(z)\left(\tilde{p}, \tilde{p}^{\prime}\right)=I(z)\left(\tilde{p}, \tilde{p}^{\prime}\right) H^{-1}\left(z-U-\frac{\tilde{p}^{\prime 2}}{2 v}\right) \\
t_{21}(z)\left(p, q ; \tilde{p}^{\prime}\right)=\frac{\lambda}{\sqrt{2}} \delta^{3}\left(p_{1}-\tilde{p}^{\prime}\right)+\frac{\lambda}{\sqrt{2}} \delta^{3}\left(p_{2}-\tilde{p}^{\prime}\right) \\
+\frac{\lambda}{\sqrt{2}} \frac{t_{11}(z)\left(p_{1}, \tilde{p}^{\prime}\right)}{H\left(z-U-\frac{p_{1}^{2}}{2 v}\right)}+\frac{\lambda}{\sqrt{2}} \frac{t_{11}(z)\left(p_{2}, \tilde{p}^{\prime}\right)}{H\left(z-U-\frac{p_{2}{ }^{2}}{2 v}\right)} \\
t_{22}(z)\left(p, q ; p^{\prime}, q^{\prime}\right)=\frac{\lambda}{\sqrt{2}} \frac{t_{12}(z)\left(p_{1} ; p^{\prime} q^{\prime}\right)}{H\left(z-U-\frac{p_{1}^{2}}{2 v}\right)}+\frac{\lambda}{\sqrt{2}} \frac{t_{12}(z)\left(p_{2} ; p^{\prime}, q^{\prime}\right)}{H\left(z-U-\frac{p_{2}^{2}}{2 v}\right)}
\end{array}
$$


Graphically

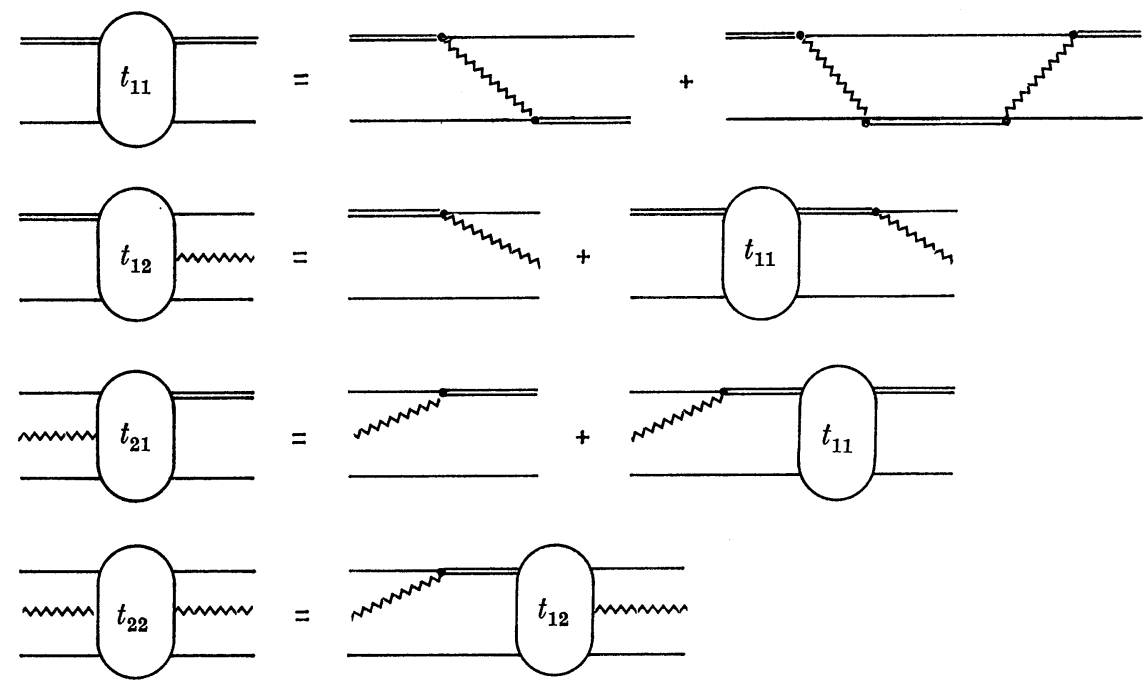

三 describes the renormalized propagator of a noninteracting $V$ and $\Theta$ particle, $\overline{\underline{m}}$ the propagator of two $\Theta$ particles and one $N$ particle. The symmetry conditions have not been taken into account. Each vertex corresponds to a multiplication by $\lambda_{0}$ and momenta are conserved. Integration is carried out over all closed loops. $\lambda_{0}=Z_{1}^{-1 / 2} \lambda$ has been used. $t(z)$ is the scattering amplitude. Scattering processes with 3 particles in the initial or final channel contain factors describing initial- or final-state interactions [6, 15].

Summing (28) gives the Källén-Pauli equation [4]

$$
t_{11}(z)\left(\tilde{p}, \tilde{p}^{\prime}\right)=I(z)\left(\tilde{p}, \tilde{p}^{\prime}\right)+\int K(z)\left(\tilde{p}, \tilde{p}^{\prime \prime}\right) t_{11}(z)\left(\tilde{p}^{\prime \prime}, \tilde{p}^{\prime}\right) d^{3} \tilde{p}^{\prime \prime} .
$$

$K(z)$ is a Hilbert-Schmidt operator in $\mathscr{L}^{2}\left(\mathbb{R}^{3}\right)$, analytic in $G_{0+}$ $=\{z \mid d(z)>0\}$ and the Hilbert-Schmidt norm satisfies

$$
\lim _{d(z) \rightarrow \infty}\|K(z)\|=0 \text {. }
$$

Also $\sup _{\tilde{z}^{\prime}}\left\|I(z)\left(\cdot, \tilde{p}^{\prime}\right)\right\|_{2}<\infty$, thus the above series (28) converges for sufficiently large $d(z)$ uniformly in $\tilde{p}^{\prime}$. Furthermore since $(1-K(z))^{-1}$ exists for sufficiently large $d(z)$, by a theorem of RELLrCH ([14], Chap. VII, [16]) $(1-K(z))^{-1}$ is meromorphic in $G_{0+}$ with values in the set of all bounded linear operators in $\mathscr{L}^{2}\left(\mathbb{R}^{3}\right)$. Some trivial estimates then show that if we define $r^{S}(z)$ by (27), (29), (30), (31) and (32) $r^{S}(z)$ becomes a bounded linear operator which is meromorphic in $G_{0+}$. By the identity theorem of analytic functions, this must then be the resolvent of $H^{S}$. Therefore it will be sufficient to discuss the Källén-Pauli equation. 
Theorem 5. For $\operatorname{Im} z \neq 0$ no nontrivial solutions of the homogeneous Källén-Pauli equation exist and (32) has therefore always a unique solution in that case.

Proof. Assume the contrary and let $\varphi$ be a solution of the homogemeous Källén-Pauli equation for $z_{0}\left(\operatorname{Im} z_{0} \neq 0\right)$ :

$$
\varphi(\tilde{p})=\int K\left(z_{0}\right)\left(\tilde{p}, \tilde{p}^{\prime}\right) \varphi\left(\tilde{p}^{\prime}\right) d^{3} \tilde{p}^{\prime} .
$$

Define $\Phi \in \mathscr{H}_{2}$ to be $(g, f)$ where

$$
\begin{aligned}
g(\tilde{p}) & =\frac{Z_{1}^{1 / 2} \varphi(\tilde{p})}{H\left(z_{0}-U-\frac{\tilde{p}^{2}}{2 v}\right)} \\
f(p, q) & =\frac{\lambda}{\sqrt{2}} \frac{1}{\left(z_{0}-\frac{p^{2}}{2 v}-\frac{q^{2}}{2 \mu}\right)}\left\{\frac{\varphi\left(p_{1}\right)}{H\left(z_{0}-U-\frac{p_{1}^{2}}{2 v}\right)}+\frac{\varphi\left(p_{2}\right)}{H\left(z_{0}-U-\frac{p_{2}^{2}}{2 v}\right)}\right\} .
\end{aligned}
$$

A lengthy calculation using (32) and (33) gives

$$
r^{S}(z) \Phi=\frac{1}{z-z_{0}} \Phi,
$$

$d(z)$ sufficiently large. But then $\Phi$ would be an eigenstate of $H^{S}$ with eigenvalue $z_{0}$. This contradicts the selfadjointness of $H^{S}$.

Thus we only may have solutions of the homogeneous Källén-Pauli equation for $z=\tau \pm i 0$ ( $\tau$ real). Let $\Phi^{ \pm}$be the set of those $\tau$, for which $z=\tau \pm i 0$ gives nontrivial solutions of (33).

It is not hard to prove that $\left(\Phi^{+} \cup \Phi^{-}\right) \cap(-\infty, U)$ is exactly the set of poles of $r^{S}(z)$ in $G_{0+}$.

Let $\varphi_{l}^{j}(1 \leqq l \leqq N(j)<\infty)$ be a basis for the linear space of all solutions of the homogeneous Källén-Pauli equation at $z^{j}<U$. Define $\Phi_{l}^{j}$ by (34) and let $P^{j}=\lim _{z \rightarrow z_{j}}\left(z-z_{j}\right) r^{S}(z)$. Some easy calculations show that $P^{j}$ is the projection on the linear span of $\Phi_{l}{ }^{j}$.

In order to consider $K(z)$ on the cut $U \leqq z<\infty$ we apply methods, which habe been extensively used by FADDEev [11] in the three body problem:

Let $b(1+\Theta, \mu)$ be the linear space of all Hölder continuous functions no $\mathbb{R}^{3}$ of index $\mu$ such that

$$
\begin{gathered}
\|f\|_{\Theta, \mu}=\sup _{p, 0<|\Delta| \leqq 1}(1+|p|)^{1+\Theta}\left\{f(p)+\frac{|f(p+\Delta)-f(p)|}{|\Delta|^{\mu}}\right\}<\infty \\
0<\Theta ; \quad 0<\mu \leqq 1 ; \quad p, \Delta \in \mathbb{R}^{3} .
\end{gathered}
$$

With this norm $b(1+\Theta, \mu)$ becomes a Banach space. With respect to the inclusion $b(1+\Theta, \mu)<b\left(1+\Theta^{\prime}, \mu^{\prime}\right), \Theta>\Theta^{\prime}, \mu>\mu^{\prime}$ a bounded set in $b(1+\Theta, \mu)$ is relatively compact in $b\left(1+\Theta^{\prime}, \mu^{\prime}\right)$. Using Faddeev's estimates we proved the following ([11], page $41 \mathrm{ff}$.$) :$ 
Theorem 6. For all $z$ in the cut plane $U \leqq z<\infty$ the maps

$$
\begin{gathered}
K(z): b\left(1+\widetilde{\Theta}, \mu^{\prime}\right) \rightarrow b\left(2, \mu^{\prime \prime}\right) ; \mu^{\prime \prime}<\operatorname{Min}\left(\frac{1}{2}, \mu^{\prime}\right) ; \frac{1}{2}<\widetilde{\Theta}<1 \\
K^{4}(z): b\left(1+\widetilde{\Theta}, \mu^{\prime \prime \prime}\right) \rightarrow b(2, \tilde{\mu}) ; \tilde{\mu}<\frac{1}{8}, \quad 0<\mu^{\prime \prime \prime}<1
\end{gathered}
$$

are continuous. The following estimates hold

$$
\begin{gathered}
\|K(z) f\|_{1, \mu^{\prime \prime}} \leqq C \lambda^{2}(1+|z|)^{-\Theta^{\prime} / 4}\|f\|_{\tilde{\Theta}, \mu^{\prime}} \\
\|(K(z+\Delta)-K(z)) f\|_{1, \mu_{1}} \leqq C \lambda^{2}(1+|z|)^{-\Theta^{\prime} / 4}\|f\|_{\tilde{\Theta}, \mu^{\prime}}|\Delta|^{\mu_{2}} \\
\left\|K^{4}(z) f\right\|_{1}, \tilde{\mu} \leqq C(1+|z|)\|f\|_{\tilde{\Theta}, \mu^{\prime \prime \prime}} \\
\Theta^{\prime}<\widetilde{\Theta}, \mu_{1}+\mu_{2} \leqq \mu^{\prime \prime}, \quad \Delta \in \mathbb{C} .
\end{gathered}
$$

$C$ may be chosen independently of $z$ and $\lambda\left(0<|\lambda| \leqq \lambda_{1}<\lambda_{c}(1)\right)$.

In particular $K^{4}(z)$ may be considered as a compact operator in $b(1+\widetilde{\Theta}, \tilde{\mu})$ since the continuous image of a bounded set is bounded. Therefore Fredholm's alternative holds ([17]):

I. Either the equation

$$
f=K(z) f ; \quad f \in \hat{b}(1+\widetilde{\Theta}, \tilde{\mu})=\bigcup_{\mu^{\prime}>\tilde{\mu}} b\left(1+\widetilde{\Theta}, \mu^{\prime}\right)
$$

has a nontrivial solution or

II. $f=g+K(z) f$ has a unique solution

$$
f \in \hat{b}(1+\widetilde{\Theta}, \tilde{\mu}) \text { for } g \in \hat{b}(1+\widetilde{\Theta}, \tilde{\mu}) \text {. }
$$

If II is satisfied then $f$ depends continuously on $\left(g, K(z) g, K^{2}(z) g\right.$, $\left.K^{3}(z) g\right)$ where all elements are considered as elements of $b(1+\Theta, \mu)$. But then theorem 6 gives

$$
\|f\|_{\tilde{\Theta}, \tilde{\mu}} \leqq C^{\prime}\|g\|_{\tilde{\Theta}, \bar{\mu}} \quad \tilde{\mu}>\bar{\mu} .
$$

Let $f$ be a solution of the homogeneous equation. $f=K(z) f$ implies in particular $f=K^{12}(z) f$. The estimates of theorem 6 then give

$$
\begin{aligned}
\left\|K^{12}(z) f\right\|_{\tilde{\Theta}, \tilde{\mu}} & \leqq C^{8} \lambda^{16}(1+|z|)^{-2 \Theta^{\prime}}\left\|K^{4}(z) f\right\|_{\tilde{\Theta}, \tilde{\mu}^{\prime}} \\
& \leqq C^{9} \lambda^{16}(1+|z|)^{1-2 \Theta^{\prime}}\|f\|_{\Theta}, \tilde{\mu} \\
\tilde{\mu}^{\prime} & <\tilde{\mu} .
\end{aligned}
$$

Since $\tilde{\Theta}>\frac{1}{2}, \Theta^{\prime}$ may also be chosen $>\frac{1}{2}$. For sufficiently large $|z|$ or sufficiently small $\lambda$ there are thus no solutions of the homogeneous Källén-Pauli equation [5a].

Generally we have

Lemma 7. $\Phi^{ \pm}$are closed sets.

This is simply lemma (7.8) of FADDEEv ([11]). We will, however, assume that there exist no solutions on the cut ${ }^{1}$.

\footnotetext{
${ }^{1}$ See Appendix B for a discussion of the possible solutions on the cut.
} 
As we have seen this is the case for sufficiently small $\lambda$.

In particular Lemma 7 shows that the number of poles of $r^{S}(z)$ below the cut is finite since $U$ is the only possible limit of these poles.

Now theorem 6 is not immediately applicable to $(32)$ since $I(z)\left(\cdot, \tilde{p}^{\prime}\right)$ is not in $\hat{b}(1+\widetilde{\Theta}, \tilde{\mu})$ for $z$ on the cut. We define

$$
I^{(i)}(z)\left(\cdot, \tilde{p}^{\prime}\right)=K^{i}(z) I(z)\left(\cdot, \tilde{p}^{\prime}\right) \quad i \geqq 0 .
$$

Using the same estimates as for theorem 6 we have shown that $I^{(3)}(z)\left(\tilde{p}, \tilde{p}^{\prime}\right)$ is Höldercontinuous with index $\tilde{\mu}<\frac{1}{8}$ in all variables such that

$$
\left\|I^{(3)}(z)\left(\cdot, \tilde{p}^{\prime}\right)\right\|_{\tilde{\Theta}, \tilde{\mu}}<C(1+|z|) .
$$

Defining $t_{11}^{(3)}(z)\left(\tilde{p}, \tilde{p}^{\prime}\right)=t_{11}(z)\left(\tilde{p}, \tilde{p}^{\prime}\right)-\sum_{i=0}^{2} I^{(i)}(z)\left(\tilde{p}, \tilde{p}^{\prime}\right)$ we get the following equation instead of (32):

$$
t_{11}^{\left({ }^{(3)}\right.}(z)\left(\cdot, \tilde{p}^{\prime}\right)=I^{(3)}(z)\left(\cdot, \tilde{p}^{\prime}\right)+K(z) t_{11}^{(3)}(z)\left(\cdot, \tilde{p}^{\prime}\right) .
$$

The above discussion gives

Theorem 7. $t_{11}^{(3)}(z)\left(\tilde{p}, \tilde{p}^{\prime}\right)$ is Höldercontinuous of index $\tilde{\mu}<\frac{1}{8}$ in all variables for all $z$ in the cut plane except for $z \in \Phi^{ \pm}$. The following estimates hold

$$
\begin{aligned}
& \left\|t_{11}{ }^{(3)}(z)\left(\cdot, \tilde{p}^{\prime}\right)\right\| \tilde{\Theta}, \tilde{\mu} \leqq C_{1}(|z|) \\
& \left\|t_{11}{ }^{(3)}\left(z+\Delta_{1}\right)\left(\cdot, \tilde{p}^{\prime}+\Delta_{2}\right)-t_{11}{ }^{(3)}(z)\left(\cdot, \tilde{p}^{\prime}\right)\right\| \tilde{\Theta}_{\Theta}, \tilde{\mu}_{1} \leqq C_{1}(|z|)\left[\left|\Delta_{1}\right|^{\tilde{\mu}_{2}}+\left|\Delta_{2}\right|^{\tilde{\mu}_{2}}\right] \\
& \Delta_{1} \in \mathbb{C}, \quad \Delta_{2} \in \mathbb{R}^{3}, \quad \tilde{\mu}_{1}+\tilde{\mu}_{2} \leqq \tilde{\mu} .
\end{aligned}
$$

$C_{1}(|z|)$ behaves as $(1+|z|)$ for large $|z|$.

We are now ready to discuss the scattering theory:

Let $\Delta(\tau)$ be given by

$\Delta(\tau)\left(\tilde{p}, p, q ; \tilde{p}^{\prime}, p^{\prime}, q^{\prime}\right)=\left(\begin{array}{lc}\delta\left(\tau-U-\frac{\tilde{p}^{2}}{2 \nu}\right) \delta^{3}\left(\tilde{p}-\tilde{p}^{\prime}\right) & 0 \\ 0 & \delta\left(\tau-\frac{p^{2}}{2 \nu}-\frac{q^{2}}{2 \mu}\right) \delta^{3}\left(p_{1}-p_{1}^{\prime}\right) \delta^{3}\left(q_{1}-q_{1}^{\prime}\right)\end{array}\right)$

Then $\Delta(\tau) \Delta\left(\tau^{\prime}\right)=\Delta(\tau) \delta\left(\tau-\tau^{\prime}\right)$.

Put

$$
u^{ \pm}=\int_{-\infty}^{+\infty}\left(e^{\prime}+r_{0}{ }^{R, S}(\tau \mp i 0) t(\tau \mp i 0)\right) \Delta(\tau) d \tau .
$$

$u^{ \pm}$are defined on the dense set $\mathscr{D}_{0}$ in $\mathscr{H}_{2}$ consisting of all $\Phi=(g, f)$ where $g, f$ are smooth and have compact support. Using the same arguments as FADDEEV ([11], page $60 \mathrm{ff}$.$) , (32) resp. (32') and the estima-$ tes (36) show that $u^{ \pm}$are isometric operators on $\mathscr{D}_{0}$ and therefore may be extended to isometric operators on $\mathscr{H}_{2}$. Let $r_{0}{ }^{S}(z)$ be the resolvent of the free Hamiltonian $H_{0} S(U)=H_{0}(U)-(2 \mathscr{M})^{-1} \mathscr{P}^{2}$. Then $u^{ \pm}$are intertwining operators for $H_{0} S(U)$ and $H^{S}$ and thus the standard scattering operators:

12 Commun.math. Phys., Vol.10 
Theorem 8. $u^{ \pm}$are isometric operators on $\mathscr{H}_{2}$ and the following relations hold for all $z(\operatorname{Im} z \neq 0)$ :

$$
r^{S}(z) u^{ \pm}=u^{ \pm} r_{0} S(z) .
$$

The calculations leading to (38) are lengthy but straightforward. The Källén-Pauli equation and the defining relation for the renormalization constant $Z_{1}$ have frequently been applied.

In order to prove unitarity, we start from the spectral resolution $E(\tau)$ of $H^{S}$. Let $P=\sum_{j} P^{j}$ be the (by the above assumption finite dimensional) projection on the bound states. Then $P=\int_{-\infty}^{U} d E(\tau)$.

For $\Phi_{1}, \Phi_{2} \in \mathscr{D}_{0}$

$$
\int_{\tau_{1}}^{\tau_{2}} d\left(\Phi_{1}, E(\tau) \Phi_{2}\right)=\frac{1}{2 \pi i} \lim _{\varepsilon \downarrow 0} \int_{\tau_{1}}^{\tau_{2}}\left(\Phi_{1}, r^{S}(\tau-i \varepsilon)-r^{S}(\tau+i \varepsilon) \Phi_{2}\right) d \tau .
$$

Theorem 9. For $\tau \geqq U, \Phi_{1}, \Phi_{2} \in \mathscr{D}_{0}$

In particular

$$
\begin{aligned}
\lim _{\varepsilon \downarrow 0} \frac{1}{2 \pi i}\left(\Phi_{1}, r^{S}(\tau-i \varepsilon)-r^{S}(\tau+i \varepsilon) \Phi_{2}\right) \\
\quad=\lim _{\varepsilon \downarrow 0} \frac{\varepsilon}{\pi}\left(\Phi_{1}, r^{S}(\tau+i \varepsilon) r^{S}(\tau-i \varepsilon) \Phi_{2}\right) \\
=\lim _{\varepsilon \downarrow 0} \frac{\varepsilon}{\pi}\left(\Phi_{1}, r^{S}(\tau-i \varepsilon) r^{S}(\tau+i \varepsilon) \Phi_{2}\right) \\
=\left(\Phi_{1}, u^{ \pm} \Delta(\tau)\left(u^{ \pm}\right)^{\dagger} \Phi_{2}\right) .
\end{aligned}
$$

$$
u^{ \pm}\left(u^{ \pm}\right)^{\dagger}=\mathbf{1}-P .
$$

For the proof the defining relation (27) must be used. In contrast to FADDEEV ([11], page 63) however we may not take the isolated limit

$$
\lim _{\varepsilon \downarrow 0} \frac{\varepsilon}{\pi} r_{0}^{R, S}(\tau+i \varepsilon) r_{0}^{R, S}(\tau-i \varepsilon) .
$$

The reason is that $t(z)$ is not sufficiently smooth, i. e. $t(z)$ contains $\delta$-functions in the momenta.

(39) finally allows us to consider the time-dependent behaviour. Let

$$
\begin{aligned}
V^{*}(P)= & Z_{1}{ }^{1 / 2} V^{*}(P) \\
& +\lambda \int \frac{1}{U-\omega} N^{*}\left(\frac{m_{3}}{m_{1}} P+q\right) \Theta *\left(\frac{m_{2}}{m_{1}} P-q\right) d^{3} q .
\end{aligned}
$$

Suppose $g(P, \tilde{p})$ and $f\left(P, p_{1}, q_{1}\right)=f\left(P, p_{2}, q_{2}\right)$ are smooth with compact support $\left(P, p_{1}, q_{1}, p_{2}, q_{2}\right.$ were defined in (26)). 
Theorem 10.

$$
\begin{gathered}
\lim _{t \rightarrow \pm \infty} e^{i H t} e^{-i H_{0}(U) t} \int g(P, \tilde{p}) \Theta^{*}\left(\frac{m_{2} P}{m_{1}+m_{2}}-\tilde{p}\right) \\
\cdot V^{*}\left(\frac{m_{1}}{m_{1}+m_{2}} P+\tilde{p}\right) \Omega d^{3} P d^{3} \tilde{p} \\
=Z_{1}^{1 / 2} \int g(P, \tilde{p})|V \Theta(P, \tilde{p})\rangle^{ \pm} d^{3} P d^{3} \tilde{p}, \\
\lim _{t \rightarrow \pm \infty} e^{i H t} \int g(P, \tilde{p}) e^{-i\left(\frac{P^{2}}{2\left(m_{1}+m_{2}\right)}+U+\frac{\tilde{p}^{2}}{2 v}\right) t} \Theta^{*}\left(\frac{m_{2} P}{m_{1}+m_{2}}-\tilde{p}\right) \\
\cdot V_{1}^{*}\left(\frac{m_{1}}{m_{1}+m_{2}} P+\tilde{p}\right) \Omega d^{3} P d^{3} \tilde{p} \\
=\int g(P, \tilde{p})|V \Theta(P, \tilde{p})\rangle \pm d^{3} P d^{3} \tilde{p}, \\
\lim _{t \rightarrow \pm \infty} e^{i H t} e^{-i H_{0}(U) t} \int f(P, p, q) N^{*}(l) \Theta^{*}\left(k_{1}\right) \Theta^{*}\left(k_{2}\right) \Omega d^{3} k_{1} d^{3} k_{2} d^{3} l \\
=\int f(P, p, q)|N \Theta \Theta(P, p, q)\rangle^{ \pm} d^{3} P d^{3} p d^{3} q .
\end{gathered}
$$

The scattering states $|\cdot\rangle^{ \pm}$are defined according to (37).

\section{Conclusions}

The Galilean invariant Lee model has a ghostless Hamiltonoperator in each sector even in the limit of local interaction. The main reason is the energy-momentum relation in the nonrelativistic case: $E=(2 m)^{-1} p^{2}$ $+U$. This has also been used by E. NeLson [18] in the case of the scalar field with recoil.

Apart from the not completely solved problem connected with possible bound states in the continuum, we also obtained a satisfactory scattering theory in the lowest sectors $\mathscr{H}(1,1), \mathscr{H}(2,1)$ and $\mathscr{H}(1,2)$. For the higher sectors we encounter the same problems as in the multiparticle case of potential scattering. To study $H$ in $\mathscr{H}\left(N_{1}, N_{2}\right)$ $\left(N_{1}, N_{2} \geqq 2\right)$ it would first be necessary to set up FADDEEV-YAKUBOWSKI ([19]) equations.

Acknowledgements. It is a pleasure to thank Prof. K. HEPP for his impelling interest in this work. Also I would like to thank Prof. M. Frenz and Prof. R. JosT giving me the opportunity to carry out this work at the Seminar für theoretische Physik der E. T. H. in Zürich.

\section{Appendix A}

We give a short discussions of $H(\chi, z)$ :

Put

$$
G(\chi, z)=Z_{\chi}+\lambda^{2} \int \frac{\chi^{2}(\omega) d^{3} q}{(U-\omega)(U-\omega+Z)}
$$

then $H(\chi, z)=z G(\chi, z) . G(\chi, z)$ is still defined for $\chi \equiv 1$. Denote the corresponding functions by $G(z)$ and $H(z)$. $G(z)$ may explicitly be $12^{*}$ 
calculated

$$
\begin{aligned}
G(z) & =1-\lambda_{c}^{-2}(1) \lambda^{2}+\frac{2 \lambda(1)_{c}^{-2} \lambda^{2}(-U)^{1 / 2}}{(-U)^{1 / 2}+(-U-z)^{1 / 2}} \\
\lambda_{c}(1) & =\frac{1}{\pi^{2}}\left(\frac{-U}{2 \mu^{3}}\right)^{1 / 2} .
\end{aligned}
$$

Generally $G(\chi, 0)=1, G(\chi, \infty)=Z_{\chi} . G(\chi, z)$ is an analytic function in the cut plane $-U \leqq z<\infty$.

If we set

$$
\begin{aligned}
\operatorname{Im} G(\chi, \tau) & =\lambda^{2} 4 \pi^{2} \tau^{-1}\left(2 \mu^{3}(\tau+U)\right)^{1 / 2} \chi^{2}(\tau+U) \\
\tau & >-U
\end{aligned}
$$

we get the following dispersion relations [10]

$$
\begin{aligned}
G(\chi, z) & =Z_{\chi}+\frac{1}{\pi} \int_{-U}^{\infty} \frac{\operatorname{Im} G(\chi, \tau)}{\tau-z} d \tau \\
& =1+\frac{z}{\pi} \int_{-U}^{\infty} \frac{\operatorname{Im} G(\chi, \tau)}{\tau(\tau-z)} d \tau \\
H^{-1}(\chi, z) & =\frac{1}{z}+\frac{1}{\pi} \int_{-U}^{\infty} \operatorname{Im} \frac{1}{H(\chi, \tau)} \frac{d \tau}{\tau-z} .
\end{aligned}
$$

This gives another expression for $Z_{\chi}$ :

$$
Z_{\chi}^{-1}=1+\lambda^{2} \int \frac{\chi^{2}(\omega)}{|H(\chi, \omega-U)|^{2}} d^{3} q .
$$

\section{Appendix B}

We want to make some remarks about possible solutions of the homogeneous Källén-Pauli equation on the cut. Let $\varphi(\tilde{p})$ be a solution of the homogeneous Källén-Pauli equation for $z_{0}=\tau_{(-)}^{ \pm} i 0, \tau \in(U, 0)$. Then it may be proved ([11], page $21 \mathrm{ff}$.): $\varphi(\tilde{p})$ is Hölder-continuous of index $\mu>1 / 2$ and

$$
\int|\varphi(\tilde{p})|^{2} \delta\left(\tau-U-\frac{\tilde{p}^{2}}{2 v}\right) d^{3} \tilde{p}=0 .
$$

Defining $\Phi$ by (34), $\Phi$ therefore becomes an element of $\mathscr{H}_{2}$ and $r^{S}(z) \Phi$ $=(z-\tau)^{-1} \Phi(\operatorname{Im} z \neq 0)$ i. e. $\Phi$ is an eigenstate of $H^{S}$ with eigenvalue $\tau$. (B 1) further shows that the set of solutions of the homogeneous KällénPauli equation for $\tau+i 0$ and $\tau-i 0$ coincide. Furthermore $\left(\Phi^{+} \cup \Phi^{-}\right) \cap(U, 0)$ is countable and the only possible limit points are $U$ and 0 ([11], page 58). For $\tau>0$, i. e. above the treshold for the inelastic process $V \Theta \rightarrow N \Theta \Theta$, the above arguments no longer work. However, one then hopes to be able to discuss the Källén-Pauli equation by making a contour deformation of the integral. 
If we assume that $\Phi^{ \pm}$is nowhere dense in $[0, \infty)$ the isometry of $u^{ \pm}$ may still be proved. The dense domain of definition $\mathscr{D}_{0}{ }^{\prime}$ for $u^{ \pm}$is then defined to consist of elements $(g, f) \in \mathscr{D}_{0}$ such that ([11], page 69)

$\left.\begin{array}{l}\text { 1) } g(\tilde{p})=0 \text { in a neighborhood of } U+\frac{\tilde{p}^{2}}{2 v}=\tau \\ \text { 2) } f(p, q)=0 \text { in a neighborhood of } \frac{p^{2}}{2 v}+\frac{q^{2}}{2 \mu}=\tau\end{array}\right\} \tau \in \Phi^{+} \cup \Phi^{-}$.

Unitarity may also be proved: We split the spectral function $E(\tau)$ into a continuous part $E_{c}(\tau)$ and a step function $E_{d}(\tau)$. Although we could not prove that all solutions of the homogeneous Källén-Pauli equation define eigenstates of $H^{S}$, it is clear that the set of "Sprungpunkte" [21] belongs to $\Phi^{+} \cup \Phi^{-}$because of theorem 7. For $\Phi_{1}, \Phi_{2} \in \mathscr{D}_{0}^{\prime}$ we then have

$$
\frac{d}{d \tau}\left(\Phi_{1}, E_{c}(\tau) \Phi_{2}\right)=\left(\Phi_{1}, u^{ \pm} \Delta(\tau)\left(u^{ \pm}\right)^{\dagger} \Phi_{2}\right) .
$$

Putting $P_{d}=\int_{-\infty}^{+\infty} d E_{d}(\tau)$ we now obtain

$$
u^{ \pm}\left(u^{ \pm}\right)^{\dagger}=\mathbf{1}-P_{d} .
$$

\section{Literature}

1. Levy-Leblond, J. M.: Commun. Math. Phys. 4, 157 (1967).

2. Bargmann, V.: Ann. Math. 59, 1 (1954).

3. Lee, T. P.: Phys. Rev. 95, 1329 (1954).

4. KäLlÉn, G., and W. PaUli: Dan. Mat. Fys. Medd. 30, Nr. 7 (1955).

5a. Pagnamenta, A.: J. Math. Phys. 6, 955 (1965); 7, 356 (1966).

5b. Kenschaft, R. P., and R. D. Amado: J. Math. Phys. 5, 1340 (1964).

5c. Sommerfield, C. M.: J. Math. Phys. 6, 1170 (1965).

5d. Kazes, E.: J. Math. Phys. 6, 1772 (1965).

6a. Maxon, M. S., and R. B. Curtis: Phys. Rev. 137, 996 (1965).

6b. - University of California preprint UCRL-14820 (1966).

7a. Goldberger, M. L., and S. B. Treiman: Phys. Rev. 113, 1636 (1959).

7 b. De Celles, P., and O. Feldmann : Nucl. Phys. 14, 517 (1960).

7c. Amado, R. D.: Phys. Rev. 122, 696 (1961).

8a. Hamermesh, M.: Ann. Phys. 9, 518 (1960).

8b. Levy-Leblond, J. M.: J. Math. Phys. 4, 776 (1963).

8c. Schrader, R.: Diplomarbeit, Hamburg (1964); unpublished.

9. Kato, T.: Trans. Am. Math. Soc. 70, 195 (1951).

10a. HAAG, R.: Phys. Rev. 112, 669 (1958).

10b. Ruelle, D.: Helv. Phys. Acta 35, 147 (1962).

11. FadDeev, L. D.: Mathematical aspects of the threebody problem in the quantum scattering theory (Transl. f. russian). Israel program for scientific translations, Jerusalem, 1965.

12. Weinberg, S.: Phys. Rev. 133B, 232 (1964).

13. Hille, E., and R. S. PhIllIPs: Functional analysis and semigroups. Providence: Am. Math. Soc. 1957. 
14. Dunford, N., and J. T. Schwartz: Linear operators I., II. New York: Interscience 1963.

15. VAUGHN, M. F.: Nuovo Cimento 40, 803 (1965).

16. Hunziker, W.: Helv. Phys. Acta 39, 451 (1966).

17. Albeverio, S., W. Hunziker, W. Sohneider, and R. Schrader: Helv. Phys. Acta 40, 745 (1967).

18. Nelson, E.: J. Math. Phys. 5, 1190 (1964).

19. YaKUBOWSKI, O. A.: Nuclear physics (in Russian) 5, 1312 (1967).

20. Levy, M.: Nuovo Cimento 13, 115 (1959).

21. Achieser, N. I., and I. M. GlasmanN: Theorie der lin. Operatoren im Hilbertraum (Transl. f. russian). Berlin: Akademie Verlag 1965.

R. Schrader

Seminar für theoret. Physik der Eidgen. Techn. Hochschule

CH 8044 Zürich, Hochstr. 60 